\title{
Avaliação comparativa e evolutiva dos protocolos de atendimento dos pacientes fissurados
}

\author{
Comparative and evolutive evaluation of attendance protocols of patients with \\ clef lip and palate
}

\author{
Nivaldo Alonso ${ }^{1}$ \\ Daniela Yukie SAKai \\ TANIKAWA $^{2}$ \\ Jonas ERALDO DE Lima \\ JuNIOR $^{3}$ \\ Marcus Castro Ferreira ${ }^{4}$
}

Trabalho realizado na Disciplina de Cirurgia Plástica da Faculdade de Medicina da Universidade de São Paulo, São Paulo, SP, Brasil.

Artigo submetido pelo SGP (Sistema de Gestão de Publicações) da RBCP.

Artigo recebido: $22 / 5 / 2010$ Artigo aceito: 4/7/2010

\begin{abstract}
RESUMO
Introdução: Os protocolos de atendimento multiprofissional são tentativas para obtenção dos melhores resultados estéticos e funcionais possíveis no tratamento dos pacientes fissurados. Objetivo: Este estudo tem por objetivo analisar comparativamente os resultados obtidos no primeiro e no segundo ano após a implantação do protocolo de atendimento multiprofissional, buscando evidenciar as diferenças significativas encontradas após maior tempo de experiência desta padronização terapêutica. Método: Estudo prospectivo, 102 pacientes portadores de fissura labiopalatina foram operados no período de 12 meses, conforme protocolo prédeterminado. Os resultados obtidos foram analisados e comparados com os encontrados no ano de 2008. Resultados: O número de pacientes atendidos e cirurgias realizadas aumentou $13 \%$. Oito categorias cirúrgicas foram relatadas, palatoplastia $(35,18 \%)$ foi a mais realizada, seguida por queiloplastia (unilateral e bilateral), mantendo o mesmo padrão de distribuição. Os procedimentos secundários persistiram com altos índices (aproximadamente $30 \%$ ), porém o número de complicações diminuiu (nenhuma fístula). Conclusão: A padronização do tratamento dos pacientes fissurados demonstrou melhora nos resultados encontrados, mesmo em curto período de tempo. Entretanto, análises sistemáticas dos anos seguintes serão realizadas com o intuito de obter melhores parâmetros para o tratamento ideal.
\end{abstract}

Descritores: Fissura palatina. Fenda labial. Guia de prática clínica.

\section{SUMMARY}

Introduction: Multidisciplinary Attendance Protocols are attempts for attainment of the best aesthetic and functional results in the treatment of the patients with cleft lip and palate. Objectives: The present study aims to realize a comparative analysis of the outcomes found in the first and second years after the institution of the multidisciplinary attendance protocol. Methods: Prospective study, 102 patients with cleft lip and palate were operated in a 12 months period, submitted to a treatment following a pre-determined protocol. The outcomes were analyzed e compared with those results found in 2008. Results: The number of patients assisted and procedures increased $13 \%$. Eight surgical categories were related, palate repair (35.18\%) was the most utilized one, followed by lip repair (unilateral and bilateral), keeping the same distribution pattern. Secondary procedures had persisted with high rates (approximately 30\%), however the number of complications decreased (no fistulae). Conclusion: The standardization of the treatment demonstrated improvement in the found results, even in short period of time. However, systematic analysis of the following years will be realized with intention to get better parameters for the ideal treatment.

Descriptors: Cleft palate. Cleft lip. Practice guideline.

1. Livre Docente pela Faculdade de Medicina da Universidade de São Paulo (FMUSP); Professor Colaborador Responsável pelo Serviço de Cirurgia Craniofacial da Disciplina de Cirurgia Plástica da FMUSP, Membro Titular da Sociedade Brasileira de Cirurgia Plástica (SBCP).

2. Membro Titular da SBCP, Médico Colaborador e Pesquisador do Hospital das Clínicas da FMUSP.

3. Residente do Terceiro Ano do Programa de Cirurgia Plástica da FMUSP.

4. Professor Titular da Disciplina de Cirurgia Plástica da FMUSP; Chefe da Divisão de Cirurgia Plástica do Hospital das Clínicas da FMUSP. 


\section{INTRODUÇÃO}

Os obstáculos presentes no tratamento e acompanhamento dos pacientes portadores de fissura labiopalatina encontraram nos protocolos de atendimento multiprofissional uma tentativa para sua resolução e a obtenção dos melhores resultados estéticos e funcionais possíveis ${ }^{1,2}$.

Com o intuito de desvendar as falhas ocorridas e, dessa forma, buscar e obter resultados cada vez mais presumíveis, o seguimento destes pacientes em estudos longitudinais tornouse o melhor método a ser utilizado no julgamento criterioso dos resultados ${ }^{3,4}$. Assim, fica cada vez mais evidente que, independente da técnica empregada e do momento em que os procedimentos cirúrgicos são realizados, é este acompanhamento minucioso, planejado e individualizado por uma equipe experiente, que proporciona os melhores ganhos no tratamento global do paciente com fissura labiopalatina ${ }^{5-7}$.

Desde 2008, o Grupo de Cirurgia Craniofacial da Disciplina de Cirurgia Plástica e Queimaduras do HCFMUSP instituiu, por meio do protocolo multiprofissional de atendimento, a padronização das intervenções as quais os pacientes são submetidos (Tabela 1$)^{8}$.
A avaliação do primeiro ano após seu estabelecimento demonstrou diferenças de perfil epidemiológico decorrentes de possíveis falhas no encaminhamento dos pacientes aos centros de tratamento, porém evidenciou resultados homogêneos e sem necessidade de revisões cirúrgicas nos casos submetidos aos procedimentos primários após início do protocolo.

Levando em consideração o compromisso em promover a avaliação prospectiva dos pacientes submetidos ao tratamento, este estudo tem por objetivo analisar comparativamente os resultados obtidos no primeiro e no segundo ano após a implantação do protocolo de atendimento multiprofissional, buscando evidenciar as diferenças significativas encontradas após maior tempo de experiência desta padronização terapêutica e seguimento dos pacientes portadores de fissura labiopalatina.

\section{MÉTODO}

Estudo prospectivo, onde 102 pacientes portadores de fissura labiopalatina foram operados consecutivamente no período 1 de janeiro a 31 de dezembro de 2009, submetidos a

Tabela 1 - Protocolo do Grupo de Cirurgia Craniofacial da Disciplina de Cirurgia Plástica e Queimaduras do HCFMUSP para tratamento dos pacientes de fissuras labiopalatinas.

\begin{tabular}{ll}
\hline Idade & Procedimento e/ou avaliação \\
\hline Nascimento & Orientação quanto à amamentação. \\
& Avaliação multidisciplinar inicial: fonoaudiólogo, geneticista, otorrinolaringologista e \\
& cirurgião plástico. \\
& Início do acompanhamento da curva de crescimento. \\
& Queiloplastia: \\
& Unilateral - técnica de Millard modificada9 com rinoplastia primária estendida; \\
& Bilateral - técnica de Millard em tempo único. Se amplas - inicialmente adesão labial e, \\
após 6 meses, procedimento cirúrgico padrão. & Se otite - colocação de tubo de ventilação por otorrinolaringologista; \\
& Moldagem em gesso da fissura.
\end{tabular}

12 meses de vida

Palatoplastia segundo a técnica descrita por von Langenbeck ${ }^{4-11}$ com veloplastia intravelar ${ }^{12}$ estendida.

Início do acompanhamento anual pela equipe de fonoaudiologia.

5 anos de vida

Faringoplastia com técnica de pedículo superior nos pacientes portadores de insuficiência velofaríngea.

Nasofibroscopia e polissonografia pré e pós-operatório de 1, 6 e 12 meses, após anual. Moldagem de gesso e cefalometrias anuais.

6 anos de vida

8 a 12 anos de vida

13 a 15 anos de vida

$>15$ anos
Início do acompanhamento odontológico.

Enxerto ósseo alveolar com osso proveniente da crista ilíaca.

Avaliação por meio de radiografia panorâmica, oclusal e periapical; e tomografia computadorizada da face pré e pós-operatório de 6 a 12 meses.

Cirurgia ortognática - se hipoplasia do terço médio da face.

Rinoplastia secundária, quando necessária. 
tratamento realizado pelo Grupo de Cirurgia Craniofacial da Disciplina de Cirurgia Plástica e Queimaduras do HCFMUSP. Durante o período do estudo, todos os pacientes foram submetidos ao protocolo de atendimento multiprofissional instituído no ano de 2008, considerando individualmente o momento terapêutico em que os mesmos se encontravam.

Os pacientes foram analisados de acordo com a localização da deformidade, suas características morfológicas e o tipo de procedimento realizado. As cirurgias primárias e secundárias foram identificadas e analisadas separadamente.

Os resultados obtidos foram analisados e comparados com os encontrados no ano de 2008, estudo este realizado com a mesma metodologia em questão no primeiro ano após implantação do protocolo de atendimento.

\section{RESULTADOS}

Durante o período de um ano, 102 pacientes foram submetidos a procedimentos cirúrgicos, sendo 108 o número total de procedimentos realizados. Em relação aos procedimentos realizados, a grande maioria dos casos foi de palatoplastia ( 38 - 35,18\%), seguida por revisão de lábio e nariz (19 - 17,59\%), queiloplastia unilateral (18 - 16,66\%), enxerto ósseo alveolar (10 - 9,25\%), queiloplastia bilateral (9 - 8,33\%), correção de fístula $(6-5,55 \%)$, faringoplastia (3 - 2,77\%), rinoplastia ( $3-2,77 \%)$ e dois procedimentos de menor porte (alongamento de columela); sendo que seis destes procedimentos foram realizados de forma combinada. Setenta por cento das abordagens ocorreram de forma primária.

A idade média encontrada foi de 1,8 anos de idade, se considerarmos os pacientes submetidos a palatoplastia abaixo dos 6 anos de idade, com distribuição igual entre os sexos. Conforme a classificação proposta por Spina, 52,6\% dos casos apresentaram fissura palatal pós-forame incompleta. Cinquenta por cento e $31,5 \%$ dos casos foram submetidos à palatoplastia primária antes e após seis anos de idade, respectivamente, enquanto $18,5 \%$ (7 pacientes) correspondem aos casos de palatoplastia secundária (dois destes pacientes provenientes de outros centros de tratamento). Noventa e dois por cento dos pacientes foram submetidos à palatoplastia, conforme técnica descrita por Von Langenbeck associada à veloplastia intravelar estendida, porém em três casos (pacientes portadores de fissura submucosa) optou-se pela realização da veloplastia intravelar isolada (8\%).

A moldagem em gesso da fissura palatina foi realizada em todos os casos abaixo dos 6 anos de idade (Figura 1), e a média do tamanho da fenda encontrada na transição entre palato duro e mole foi de $11,3 \mathrm{~mm}$ (variando de 5 a $20 \mathrm{~mm}$ ). Durante o seguimento pós-operatório, em nenhum dos pacientes submetidos à palatoplastia constatou-se a presença de fístula completa, apenas em três casos observouse discreta deiscência do plano oral, de resolução espontânea durante a primeira semana de seguimento.

A maioria dos pacientes submetidos à queiloplastia, seja unilateral ou bilateral, era do sexo masculino $(66,7 \%)$, porém a idade média de realização do procedimento foi de cinco meses ( 3 meses - 56 anos) para a primeira e 5,1 anos ( 7 meses - 10 anos) para a segunda. Todos os pacientes portadores de fissura unilateral foram submetidos à técnica de Millard modificada com rinoplastia primária estendida, sendo que em $66,7 \%$ dos casos este procedimento ocorreu abaixo dos seis meses, considerando que obtivemos dois pacientes com idade superior a 30 anos. Quanto à distribuição do tipo de fissura encontrada, as mais comuns foram: fissura pré-forame esquerda e transforame esquerda (ambas com 33,3\% dos casos), seguidas por fissura transforame direita e pré-forame direita (ambas com $16,6 \%$ ) dos casos. Foi diagnosticada em um paciente, deiscência parcial da linha de sutura do retalho cutâneo, tratada conservadoramente. Nove pacientes, a maioria constituída de portadores de fissura transforme bilateral $(55,5 \%)$, foram submetidos à queiloplastia bilateral. $\mathrm{O}$ tempo definitivo da técnica de Spina foi utilizado em cinco casos, devido à realização prévia do primeiro e segundo tempo desta técnica. Em dois pacientes, utilizou-se a técnica de Spina em tempo único e a técnica de Millard foi empregada em um caso.

Realizou-se enxerto ósseo alveolar em dez pacientes, com idade entre oito e 14 anos (idade média $=11,7$ anos). Sessenta por cento e $40 \%$ dos casos eram secundários e terciários, respectivamente. A distribuição das fissuras seguiu a seguinte proporção: $40 \%$ transforame direita, $20 \%$ transforame esquerda, $20 \%$ pré-forame direita, $10 \%$ pré-forame esquerda e $10 \%$ transforame bilateral. Dois casos apresentaram complicações, em um deles houve exposição óssea, e no segundo, infecção da área doadora.

Faringoplastia foi realizada em três pacientes, sendo um deles provenientes de outro serviço. Três pacientes foram submetidos a rinoplastia, sendo duas unilaterais e uma bilateral.

Foram necessários procedimentos de revisão de lábio e nariz em 19 pacientes, sendo indicada revisão completa em $15,9 \%$ dos casos, e uma revisão mais ampla em $73,7 \%$ destes. Um paciente foi submetido à confecção de retalho de Abbé e outro, a pequena revisão da mucosa. Foram realizadas seis correções de fístulas, sendo quatro destes pacientes provenientes de outros centros de tratamento.

\section{DISCUSSÃO}

Ao se instituir um protocolo de atendimento, nossas expectativas em relação aos resultados obtidos aumentam a cada ano que se passa. Analisar, comparar e interpretar os sucessos conquistados com o tempo, bem como reconhecer os erros e repará-los, torna-se um exercício de perseverança e esforço, visando sempre o melhor para o paciente.

Ao comparar os dados encontrados neste estudo com os do primeiro ano do protocolo de atendimento $(2008)^{8}$, algumas diferenças consistentes foram evidenciadas, mesmo tendo ciência do curto período de tempo entre as duas avaliações, principalmente quando consideramos o tratamento global dos pacientes com fissura labiopalatina. Houve aumento aproximado de $13 \%$ 


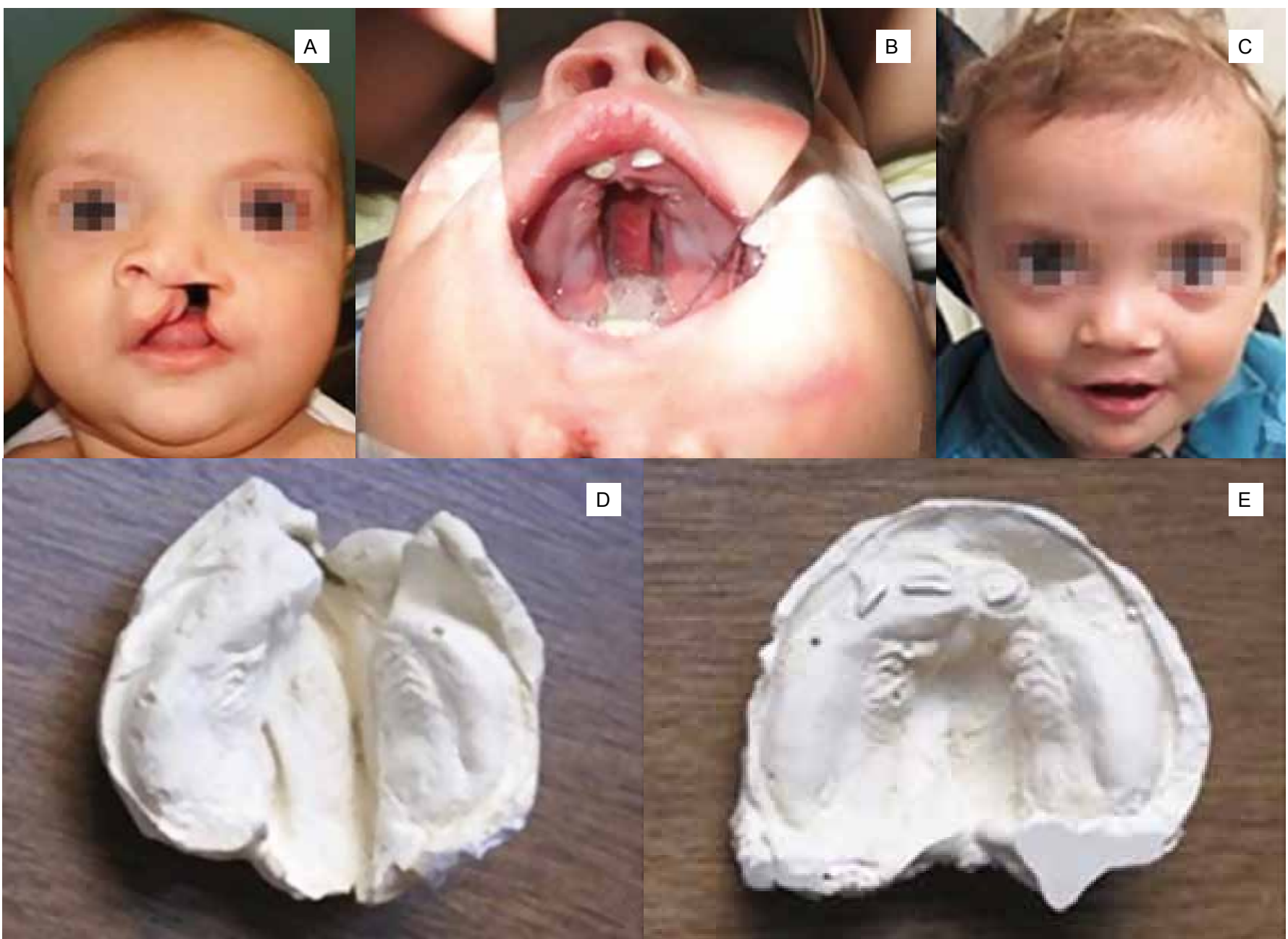

Figura 1 - Fissura transforame esquerda. A: Pré-operatório de queiloplastia. B: Pré-operatório de palatoplastia. C: Pós-operatório de queiloplastia e palatoplastia. D: Molde pré-operatório de queiloplastia. E: Molde pré-operatório de palatoplastia.

do número total de pacientes atendidos e procedimentos realizados (Tabela 2). Acreditamos que isto seja o reflexo do estabelecimento de um protocolo organizado e rigoroso quanto aos seus resultados, que oferecendo tratamento de melhor qualidade fez com que outros centros de saúde encaminhassem um número maior de pacientes a este serviço. Dessa maneira, os pacientes foram recebidos de forma mais precoce, o que consequentemente contribuiu para que o tratamento cirúrgico fosse realizado na idade mais apropriada estabelecida no protocolo em questão.

Neste segundo ano de experiência, pudemos observar que não houve diferença significativa quanto à distribuição das cirurgias realizadas, aos tipos de fissuras e suas localizações, em relação ao ano anterior. Entretanto, quando analisamos o perfil epidemiológico, encontramos maior número de pacientes adultos sem qualquer tratamento prévio, e isto pode ser considerado outra consequência relacionada ao reconhecimento deste serviço como local adequado e seguro no tratamento destes pacientes, que até então não tiveram a chance de obter assistência em outros centros.
Alguns obstáculos se mantiveram no segundo ano em que o protocolo foi desenvolvido. Considerando que muitos pacientes já eram direcionados ao Hospital das Clínicas para iniciar ou mesmo continuar seu tratamento, muitas vezes portadores de complicações e/ou sequelas, o número de procedimentos secundários permaneceu elevado (em torno de $30 \%$ ). A maioria destes procedimentos foi realizada em pacientes submetidos a cirurgia primária em outros serviços, e o restante em doentes tratados previamente à instituição da padronização dos procedimentos. O número de casos de enxertos ósseos alveolares permaneceu baixo, e isso é decorrente da dificuldade em obter preparação e acompanhamento ortodôntico adequado em nosso serviço, devida a grande demanda de pacientes e número restrito de profissionais.

Com a intenção de realizar uma avaliação global dos pacientes, possuímos em nosso Serviço protocolos para análise objetiva dos resultados estéticos (sistema de pontuação dos resultados estéticos da rinoqueiloplastia primária ${ }^{9,10,13}$ ) e de ordem funcional (função velofaríngea, oclusão dentária, 
Tabela 2 - Número de procedimentos realizados em pacientes portadores de fissura labiopalatina no ano de 2008 e 2009.

\begin{tabular}{l|c|c}
\hline Procedimentos & $\mathbf{N}^{\circ} \mathbf{( \% )}$ & $\mathbf{N}^{\circ}(\mathbf{\%})$ \\
\hline Palatoplastia & $42(41,58 \%)$ & $38(35,18 \%)$ \\
Queiloplastia unilateral & $15(14,85 \%)$ & $18(16,66 \%)$ \\
Queiloplastia bilateral & $6(5,94 \%)$ & $9(8,33 \%)$ \\
Enxerto ósseo alveolar & $10(9,90 \%)$ & $10(9,25 \%)$ \\
Faringoplastia & $2(1,98 \%)$ & $3(2,77 \%)$ \\
Rinoplastia & $7(6,94 \%)$ & $3(2,77 \%)$ \\
Revisão de lábio e nariz & $15(14,85 \%)$ & $19(17,59 \%)$ \\
Correção de fístula & $4(3,96 \%)$ & $6(5,55 \%)$ \\
Outros & - & $2(1,90 \%)$ \\
\hline Total & $101(100 \%)$ & $108(100 \%)$ \\
\hline
\end{tabular}

crescimento facial), pois na associação dos dados encontrados nestas avaliações sistemáticas poderemos encontrar as respostas para conseguir um resultado equilibrado.

De forma geral, as complicações encontradas diminuíram gradativamente de um ano para o outro. Isto pode ser visto por meio da redução importante do número de fístulas, não havendo nenhum caso registrado neste segundo ano, apesar do perfil relativo ao tamanho das fendas palatinas permanecer o mesmo. Entretanto, pequenas deiscências, sem maiores comprometimentos, estiveram presentes em poucos pacientes, nos casos operados por médicos residentes de cirurgia plástica, sob supervisão direta dos médicos assistentes responsáveis. Esse fato exemplifica o que os maiores estudos multicêntricos relatam em relação ao tratamento de pacientes fissurados, que a variável mais importante é a experiência de cirurgião ${ }^{1,5,6}$. Entretanto, nossa instituição é conhecida pelos serviços prestados e também pela sua preocupação na formação médica de qualidade. $\mathrm{O}$ treinamento adequado de médicos residentes é de extrema importância, principalmente no maior hospital escola do país, e todos compreendem haver maior necessidade na prática dos procedimentos, sem causar ônus aos pacientes.

Inicialmente, estabelecer um protocolo multidisciplinar se mostrou necessário para promover a uniformização do atendimento prestado, e dessa maneira realizar uma avaliação prospectiva fidedigna, extraindo a longo prazo as melhores experiências adquiridas e ajustando o protocolo inicial. Após a instituição da padronização do atendimento prestado, resultados positivos como os descritos anteriormente, mesmo em uma avaliação inicial de dois anos, já foram obtidos. Acreditamos que, após cinco anos de existência e seguimento rigoroso, os resultados obtidos poderão ser verificados com maior crítica, sendo também útil para realizar uma auto-avaliação quanto à qualidade dos serviços prestados por este centro de referência.

\section{CONCLUSÃO}

A padronização do tratamento dos pacientes portadores de fissura labiopalatina mediante protocolos de atendimento multidisciplinar demonstrou melhora nos resultados encontrados, mesmo em curto período de avaliação. As análises sistemáticas dos dados encontrados nos anos seguintes serão realizadas cuidadosamente, com o intuito de obter melhores resultados ao longo do tempo.

\section{REFERÊNCIAS}

1. Shaw WC, Brattström V, Molsted K, Prahl-Andersen B, Roberts CT, Semb G. The Eurocleft study: intercenter study of treatment outcome in patients with complete cleft lip and palate. Part 5: discussion and conclusions. Cleft Palate Craniofac J. 2005;42(1):93-8.

2. Robin NH, Baty H, Franklin J, Guyton F, Mann J, Woolley AL, et al. The multidisciplinary evaluation and management of cleft lip and palate. South Med J. 2006;99(10):1111-20.

3. Strong EB, Buckmiller LM. Management of the cleft palate. Facial Plast Surg Clin North Am. 2001;9(1):15-25.

4. Vander Kolk CA. Cleft palate. In: Vander Kolk CA, ed. Plastic surgery: indications, operations and outcomes. Vol. 2. Craniomaxillofacial, cleft, and pediatric surgery. St. Louis: Mosby;2000. p.799-812.

5. Brattström V, Molsted K, Prahl-Andersen B, Semb G, Shaw WC. The Eurocleft study: intercenter study of treatment outcome in patients with complete cleft lip and palate. Part 2: craniofacial form and nasolabial appearance. Cleft Palate Craniofac J. 2005;42(1):69-77.

6. Molsted K, Brattström V, Prahl-Andersen B, Shaw WC, Semb G. The Eurocleft study: intercenter study of treatment outcome in patients with complete cleft lip and palate. Part 3: dental arch relationships. Cleft Palate Craniofac J. 2005;42(1):78-82.

7. Semb G, Brattström V, Mølsted K, Prahl-Andersen B, Zuurbier P, Rumsey N, et al. The Eurocleft study: intercenter study of treatment outcome in patients with complete cleft lip and palate. Part 4: relationship among treatment outcome, patient/parent satisfaction, and the burden of care. Cleft Palate Craniofac J. 2005;42(1):83-92.

8. Alonso N, Tanikawa DYS, Lima Junior JE, Rocha DL, Sterman S, Ferreira MC. Fissuras labiopalatinas: protocolo de atendimento multidisciplinar e seguimento longitudinal em 91 pacientes consecutivos. Rev Bras Cir Plast. 2009;24(2):176-81.

9. Tanikawa DYS, Alonso N, Rocha DL. Modificação na rinoqueiloplastia primária do paciente com fissura labial unilateral. Rev Bras Cir Plast. 2010;25(1):30-7.

10. Tanikawa DYS, Alonso N, Rocha DL. Evaluation of primary cleft nose repair: severity of the cleft versus final position of the nose. J Craniofac Surg. (no prelo)

11. Cutting C, Rosembaum J, Rovati L. The technique of muscle repair in the soft palate. Oper Tech Plast Surg. 1995;2:215-22.

12. Marsh JL, Grames LM, Holtman B. Intravelar veloplasty: a prospective study. Cleft Palate J. 1989;26(1):46-50.

13. Mars M, Plint DA, Houston WJ, Bergland O, Semb G. The Goslon Yardstick: a new system of assessing dental arch relationships in children with unilateral clefts of the lip and palate. Cleft Palate J. 1987;24(4):314-22. 